\title{
Structural Studies of Self-
}

Assembled Subviral Particles:

Combining Cell-Free Expression

with $110 \mathrm{kHz}$ MAS NMR

Spectroscopy

\section{Journal Article}

\section{Author(s):}

David, Guillaume; Fogeron, Marie-Laure; Schledorn, Maarten; Montserret, Roland; Haselmann, Uta; Penzel, Susanne; Badillo, Aurélie; Lecoq, Lauriane; André, Patrice; Nassal, Michael; Bartenschlager, Ralf; Meier, Beat H.; Böckmann, Anja

\section{Publication date:}

2018-04-16

\section{Permanent link:}

https://doi.org/10.3929/ethz-b-000261015

\section{Rights / license:}

In Copyright - Non-Commercial Use Permitted

\section{Originally published in:}

Angewandte Chemie. International Edition 57(17), https://doi.org/10.1002/anie.201712091

\section{Funding acknowledgement:}

159707 - NMR studies in the Solid State (SNF)

741863 - Faster magic-angle spinning leads to a resolution revolution in biological solid-state NMR (EC) 


\section{Towards structural studies of self-assembled subviral particles: combining cell-free expression with $110 \mathrm{kHz}$ MAS NMR}

Guillaume David $^{1 /}$, Marie-Laure Fogeron ${ }^{1 /}$, Maarten Schledorn ${ }^{2 \#}$, Roland Montserret ${ }^{1}$, Uta Haselmann ${ }^{3,4}$, Susanne Penzel ${ }^{2}$, Aurélie Badillo ${ }^{1,5}$, Lauriane Lecoq ${ }^{1}$, Patrice André ${ }^{6}$, Michael $\mathrm{Nassal}^{7}$, Ralf Bartenschlager ${ }^{3,4}$, Beat H. Meier ${ }^{2 *} \&$ Anja Böckmann ${ }^{1 *}$

${ }^{1}$ Institut de Biologie et Chimie des Protéines, MMSB, Labex Ecofect, UMR 5086 CNRS, Université de Lyon, 7 passage du Vercors, 69367 Lyon, France

${ }^{2}$ Physical Chemistry, ETH Zurich, 8093 Zurich, Switzerland

${ }^{3}$ Department of Infectious Diseases, Molecular Virology, Heidelberg University, Im Neuenheimer Feld 345, 69120 Heidelberg, Germany

${ }^{4}$ Division of Virus-Associated Carcinogenesis, Germany Cancer Research Center (DKFZ), Im Neuenheimer Feld 242, 69120 Heidelberg

${ }^{5}$ RD-Biotech, Recombinant Protein Unit, 3 rue Henri Baigue, 25000 Besançon, France

${ }^{6}$ Centre International de Recherche en Infectiologie, Institut National de la Santé et de la Recherche Médicale Unité 1111, Centre National de la Recherche Scientifique Unités Mixte de Recherche 5308, Lyon, France; Ecole Normale Supérieure de Lyon, Lyon, France; Université Claude Bernard Lyon 1, Villeurbanne, France; Université de Lyon, Lyon, France; Laboratoire de Virologie, Hôpital de la Croix-Rousse, Hospices Civils de Lyon, Lyon, France

${ }^{7}$ University Hospital Freiburg, Internal Medicine II / Molecular Biology, Hugstetter Str. 55, D-79106 Freiburg, Germany

\# These authors contributed equally

For correspondence: Anja Böckmann, a.bockmann@ibcp.fr; Beat H. Meier, beme@ethz.ch 


\begin{abstract}
Viral membrane proteins are prime targets in the combat against infection. Still, their structure determination remains a challenge, both with respect to sample preparation, and the need for structural methods allowing analysis in a native-like lipid environment. Cell-free protein synthesis and solid-state NMR are promising approaches in this context, one with respect to its high potential of native expression of complex proteins, and the other for its ability to analyze membrane proteins in lipids. We here show that milligram amounts of the small envelope protein of the duck hepatitis B virus (DHBV) can be produced using cell-free expression, and that the protein self-assembles into subviral particles. 2D proton-detected NMR spectra recorded at $110 \mathrm{kHz}$ magic angle spinning on $<500 \mu \mathrm{g}$ protein show a number of isolated peaks with linewidths comparable to model membrane proteins, paving the way for structural studies of this homologous protein to a potential drug target in HBV infection.
\end{abstract}

Keywords Solid-state NMR; cell-free protein expression; duck hepatitis B virus; subviral particles; proton detection 
Infection with hepatitis B virus (HBV) entails acute or chronic diseases of the liver, the latter often associated with liver cirrhosis and hepatocellular carcinoma. HBV is a spherical enveloped virus with a $42 \mathrm{~nm}$ outer diameter ${ }^{[1]}$ that possesses a circular partially doublestranded DNA genome contained in an icosahedral capsid formed by multiple copies the core protein $(\mathrm{HBc})$. It is surrounded by a lipid envelope into which three different HBV surface proteins designated small (S), medium (M) and large (L) hepatitis B surface antigen (HBs) are embedded. One particularity of the virus is that infected cells massively produce subviral particles (SVPs), which are only composed of capsid-less (i.e. empty) virus envelopes, mainly formed by the $\mathrm{S}$ protein embedded into a phospholipid membrane derived from the host. ${ }^{[2]}$

The three viral envelope proteins $\mathrm{S}, \mathrm{M}$ and $\mathrm{L}$ differ by their N-terminal domain: in addition to $\mathrm{S}, \mathrm{M}$ carries the preS2 domain while $\mathrm{L}$ carries both the preS1 and preS2 domains. The $\mathrm{S}$ protein is the most abundant in both virion envelopes and SVPs, ${ }^{[3]}$ and can form particles on its own. ${ }^{[4]}$ The topology proposed for this protein consists of 4 transmembrane helices and a cytosolic loop as well as an antigenic loop. ${ }^{[5]}$ The protein is a potential drug target for HBV; but while topology models are available, its high-resolution structure has not been determined, as the virion envelope shows heterogeneity as observed by electron microscopy. ${ }^{[6]}$ This manifest variability suggests that in-vitro reconstituted SVPs represent an interesting alternative to virions for structural studies.

To produce the HBs S protein, we chose wheat-germ cell-free protein expression ${ }^{[7-9]}$ (Figure S1) and tested the human and duck HBs S protein in this system. Due to its fundamental similarity to $\mathrm{HBV}$, duck HBV (DHBV, see schematic representation in Figure 1a for a representation of the viral particle, as well as SVPs) is an important model for the human virus. ${ }^{[10]}$ The duck hepatitis $B$ virus $S$ protein is similar to the one of $\mathrm{HBV}$, but lacks the cysteine-rich antigenic loop of HBs S, reducing the total size of DHBs S to 167 amino acid 
residues versus 226 residues for HBs S (see Figure S2 for sequence alignments and Figure 1b for a predicted model of S). While CF HBs S expression still needs further optimization as the yields were rather low and may require the presence of chaperones to keep the protein in solution (data not shown), the DHBV protein expressed well in the presence of mild detergent and assembled spontaneously. Together with its reduced size, this feature makes DHBV SVPs a highly attractive pilot for a structural study by solid-state NMR. Importantly, the recent development of $>100 \mathrm{kHz}$ magic-angle-spinning (MAS) solid-state NMR proton detection techniques, which reduce the requested sample amount by nearly two orders of magnitude, opens the possibility to analyze the protein amounts typically obtained in cell-free expression (vide infra). While $>100 \mathrm{kHz}$ MAS NMR, ${ }^{[11-15]}$ cell-free expression, ${ }^{[16-18]}$ NMR of large assemblies ${ }^{[19,20]}$ and of membrane proteins ${ }^{[21,22]}$ have been demonstrated before, we here show that, despite the already high complexity hidden behind every single of these elements, their combination is possible and allowed us to obtain NMR data of DHBV self-assembled SVPs showing suitable resolution and sensitivity. This should lead the way for investigations of these, and similar, large membrane protein assemblies which structures remained intractable.

Cell-free expression of DHBs S was tested in small-scale bilayer reactions (see Material and Methods section in the Supporting Information), where the wheat germ extract (WGE) is overlayed with feeding buffer ${ }^{[8]}$ The expression level of the protein is good, as can be seen on the Coomassie gels and western blots in which the total reaction, as well as supernatant and pellets fractions were analyzed (Figure 1c). Expression was tested both in the absence and presence of MNG-3 detergent, which we previously identified as a powerful additive for solubilization of membrane proteins and compatible with the cell-free reaction ${ }^{[23]}$ Indeed, while in the absence of the detergent, the protein was found in the pellet, it is mainly soluble in presence of $0.1 \%$ MNG-3. 


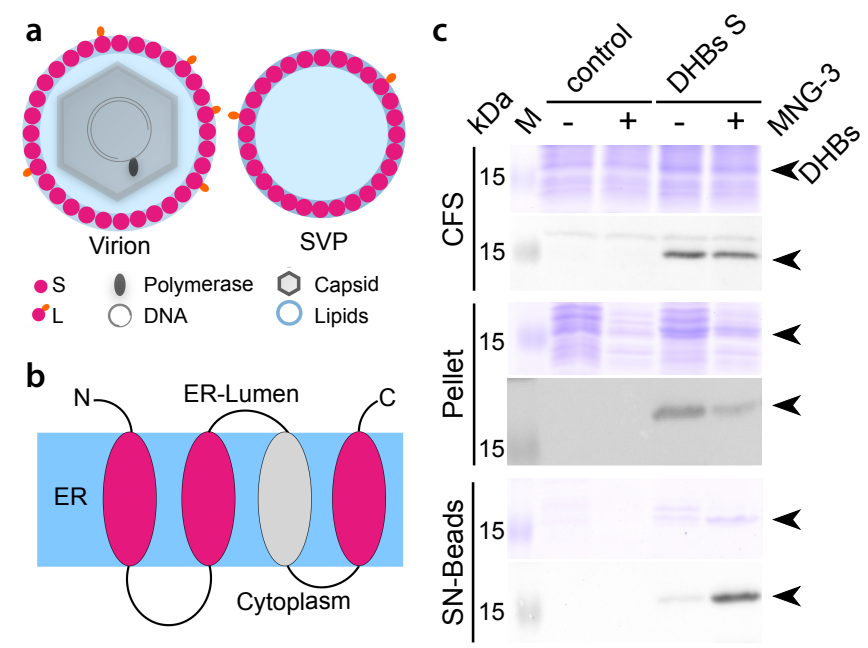

Figure 1. Expression of the small envelope protein of the DHBV virus. a Schematic representation of the DHBV virion, composed of an envelope (in pink) consisting of small (S) and large (L) surface proteins (in the human virus, a middle (M) protein exists in addition); a capsid formed by the DHBc protein (in grey), and the DNA encoding for the viral proteins (in black). The viral polymerase (black oval) is present in the virion as well. b Membrane topology model of the $\mathrm{S}$ protein, with the third helix (in grey) predicted only for the HBV S protein. ${ }^{[5,10]}$ c Expression tests of DHBs $S$ in (analytical) bilayer mode. ${ }^{[8]}$-, expression without detergent; +, expression in the presence of $0.1 \%$ MNG-3. SDS-PAGE Coomassie blue staining and Western blots using Strep-tag II antibodies are shown. CFS, total cell-free sample; Pellet, pellet; SN-beads, supernatant of CFS, incubated with Strep-Tactin magnetic beads. Comparable amounts were loaded on the gel for Pellet and SNbeads.

In order to analyze the soluble protein fractions, we loaded the supernatant from a larger reaction carried out in a dialysis cassette on a discontinuous $10-60 \%$ sucrose gradient. The protein is mainly detected in the fractions $40 \%$ and $50 \%$ sucrose fractions (Figure 2a), corresponding to a density of $1.17-1.23 \mathrm{~g} / \mathrm{mL}$ like authentic SVPs, whereas the bulk of nonrelated proteins remains mostly at the top of the gradient. The electron micrograph (EM) analysis of the $40 \%$ fraction is shown in Figure $2 \mathrm{~b}$ and displays spherical particles with a diameter of $29 \pm 3 \mathrm{~nm}$ (average measured on $\mathrm{n}=164$ particles). This homogeneity relates them better to SVPs from HBV than to the heterogeneous empty particles of $30-60 \mathrm{~nm}$ reported for DHBV. ${ }^{[24]}$ As the DHBs $\mathrm{S}$ protein self-assembles into particles and is a transmembrane protein, but no lipids are added to the reaction mixture, it is likely that these SVPs contain residual lipids present in the WGE. Indeed, when preparing WGEs, we found 
that separation of the supernatant and the lipid fraction is not quantitative on extract preparation.

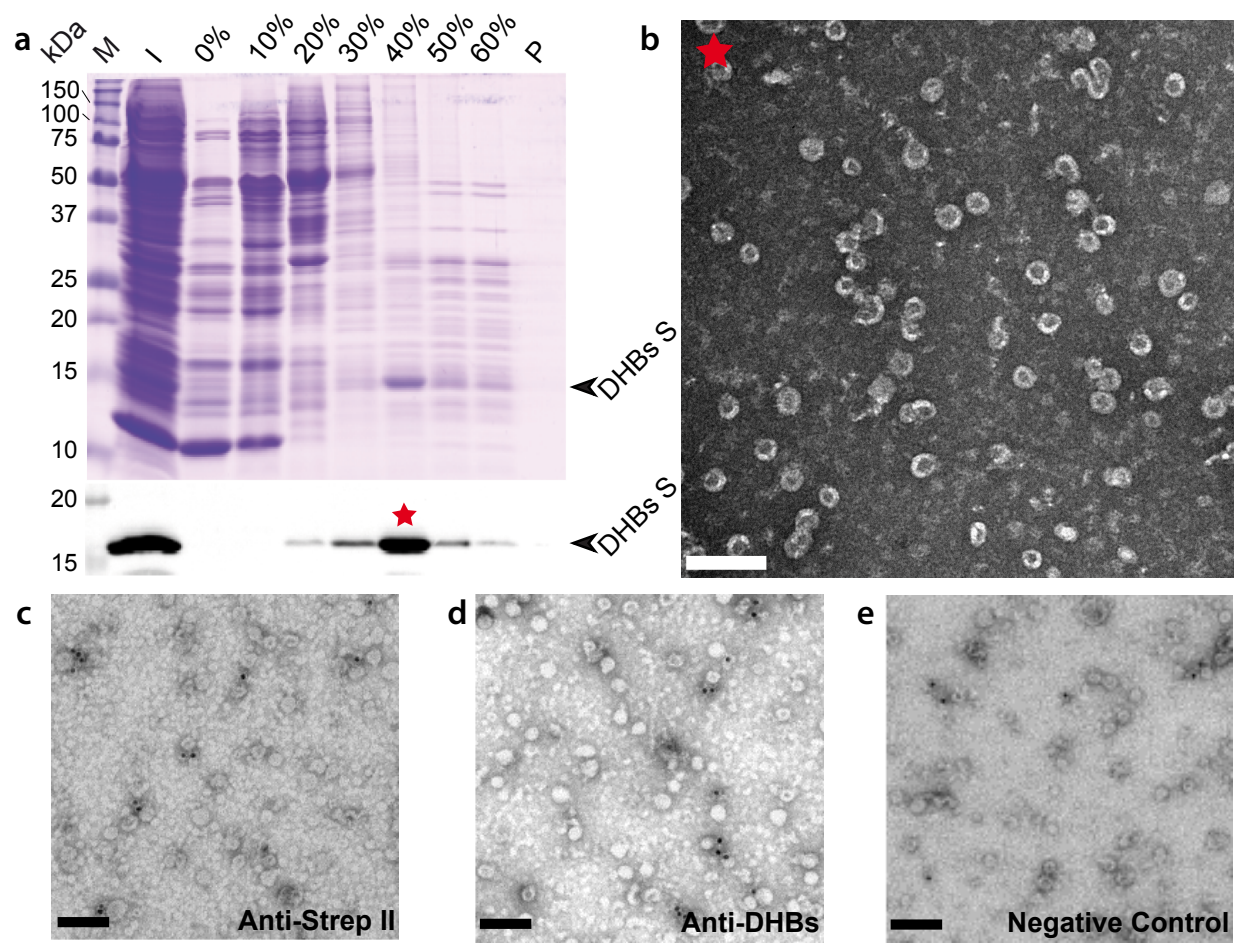

Figure 2. DHBs S self-assembles into SVP-like particles in a WGE cell-free expression system. a Analysis on a sucrose gradient of DHBs S produced in a dialysis reaction (supernatant only). SDS-PAGE (top panel) and Western blotting (bottom panel) using a Strep-tag II antibody. I, input; 10\%-60\%, sucrose concentration; P, pellet. Arrowheads indicate the protein of interest. A red star indicates the fraction used for electron microscopy (negative staining) shown in $\mathbf{b}$; scale bar, $100 \mathrm{~nm}$. c-e Transmission electron microscopy analysis of immunogold labeled DHBV SVPs using a anti Strep-tag II antibody ${ }^{[25]}(\mathbf{c})$, or a DHBs-S-specific antibody ${ }^{[26]}(\mathbf{d})$. As negative control, an anti preS-specific antibody was used (e). Scale bar $100 \mathrm{~nm}$.

To corroborate the identity and authenticity of the observed particles, we determined their antigenic properties. SVPs from the sucrose density gradient were analyzed by electron microscopy after immunogold labeling using Strep-tag II (Figure 2c) and DHBs S antigenspecific antibodies (Figure 2d). ${ }^{[26]}$ A preS specific antibody was used as a control (Figure 2e). The close proximity of the electron-dense gold particles to the SVP-like particles in c and d but not e indicates their specific recognition by anti-Strep II and anti-DHBs antibodies. The 
interaction with the anti-Strep-tag II antibody shows that the particles observed correspond indeed to particles composed by DHBs $\mathrm{S}$, and are no artefacts. The interaction with the DHBs $\mathrm{S}$ antigen-specific antibody confirms that the protein is in a state where the linear epitope against which the antibody reacts is exposed, similar as in SVPs and viral particles. ${ }^{[26]}$ In order to assess secondary structure by circular dichroism, we purified DHBs S using affinity chromatography via the Strep-tag II (Figure 3a). The CD spectra recorded on DHBs S are characteristic of a structured protein, mainly folded into $\alpha$-helices (Figure S3b) as expected from DHBs S structural models (see Figure 1b). Purification also allowed to assess expression yield, which was determined to $0.8 \mathrm{mg}$ DHBs S per mL WGE used. We also prepared a triple C48,65,69S mutant (Figure S4a), in which all cysteine residues located in the cytosolic loop are mutated to serine. This mutant does not assemble into SVPs on expression (Figure S4b) but shows, after purification (Figure S4c), a very similar profile in CD spectra (Figure S4d), indicative of a high $\alpha$-helical content, meaning that also monomers or smaller oligomers of the protein show the expected secondary structure content. As the WT protein assembles into SVPs, one can deduce that not only the helical scaffold, but also the cytosolic loop (which constitutes the major extra-helical region in the protein) in WT HBs $\mathrm{S}$ must show a conformation compatible with assembly of the protein into SVPs, indicating the functional fold of our sample. If it is perturbed, no assembly takes place.

We have prepared NMR samples of the protein using different protocols. As affinity purification resulted in particle dissociation, it was not used, and all samples had in common that the protein-containing fractions from the sucrose gradient were diluted twice and directly sedimented into the NMR rotor using dedicated filling tools. ${ }^{[27]}$ Variation of parameters included sucrose gradients, amino-acid mixtures used for expression, rotor-filling procedures and constructs. We evaluated the sample quality using 2D ${ }^{1} \mathrm{H}-{ }^{15} \mathrm{~N} 100-110 \mathrm{kHz}$ MAS solidstate NMR spectra recorded on $0.7 \mathrm{~mm}$ NMR rotors, which are shown in Figure S5. It can be 
seen there that spectrum quality depended on sample preparation; with the use of an algal amino-acid mix resulting in poor expression and signal-to-noise ratio (SNR), and with protein more efficiently filled using modified filling tools (\#1 vs \#2-4) (for details see Figure S5 caption). Whereas first trials were made using productions containing $2 \mathrm{~mL}$ of WGE, the use of $0.5 \mathrm{~mL}$ proved sufficient in the end to fill a rotor. While here home-made WGE was used ${ }^{[9]}$ resulting in costs of $650 €$ per triply labeled sample, the cost remains in the range of standard NMR samples also with commercial extracts (approx. total of $1000 €$ per sample). Figure 3 shows the NMR spectrum recorded on sample \#4 where the (unused, as for solid-state NMR sample preparation the protein is exclusively purified by the sucrose gradient centrifugation) Strep-tag II was removed from the expression plasmid. It shows the highest sensitivity (SNR $=8$ as measured on isolated signals) compared to all other preparations (Figure S5), and the 2D spectrum recorded in $19 \mathrm{~h}$ shows many distinct signals. We processed the spectrum using a $60^{\circ}$ shifted sine-bell function (SSB $=3$ in BRUKER Topspin), and plotted it just above noise level. Figure S6 shows the spectrum processed with other apodization functions, in order to illustrate the artificial resolution-enhancement that can be achieved. We measured linewidths for different signals (on a spectrum processed without resolution enhancement using a pure cosine wave, SSB = 2), and obtained values of $128-192 \mathrm{~Hz}$ for the four resolved lines marked by crosses in Figure 3. Global $\mathrm{T}_{2}$ ' times measured on series of 1D spectra were $3.9 \pm 0.6 \mathrm{~ms}$, and $\mathrm{T}_{1 \rho} 18 \pm 3 \mathrm{~ms}$ (see Table $\mathrm{S} 1$ ). Linewidths of DHBs $\mathrm{S}$ are larger than linewidths of microcrystalline proteins as ubiquitin, ${ }^{[28]} \mathrm{SH} 3^{[29]}$ or $\mathrm{GB} 1,{ }^{[30]}$ but are found close to the ones observed for deuterated, $\mathrm{HN}$ backexchanged and fully protonated model membrane proteins, as $O \mathrm{mpG}^{[29]}$ and proteorhodopsin, ${ }^{[14]}$ which show around 100 and $190 \mathrm{~Hz}$ proton linewidths respectively. While deuteration is always benefitial in terms of linewidth, it only reduces the coherent linewidth, and depending on the size of incoherent contributions, the effect can be larger or smaller. To separate the effects as much as possible, we therefore 
use only perdeuterated proteins in this study. 1D traces along different nitrogen dimensions are shown in Figure 3 to illustrate the signals in the proton dimension and their signal-to-noise ratio. The signals in the spectrum are distributed over a spectral width that can be expected for a fully $\alpha$-helical protein, which shows by nature a smaller chemical-shift dispersion than proteins containing $\beta$-strand or both $\alpha$-helical and $\beta$-strand conformation. Indeed, as shown in Figure S7, the spectral region of the backbone amide correlations in the DHBs S spectrum highly coincides with $\mathrm{NH}$ correlations simulated from BMRB chemical shifts of a mainly $\alpha-$ helical protein, here proteorhodopsin, ${ }^{[31]}$ and lacks the typically larger distribution of a $\beta$ barrel protein chemical shifts, as OmpX.$^{[32]}$ Besides the backbone NH correlations, one sidechain signal is observed for His60 (only His in the protein, located in the cytosolic loop) and three or four for Trp (total of 7), with dispersed chemical shifts due to sequence-specific environments. The signal in the upper left corner likely stems from an Arg side chain (three total), which shows an outlier $\mathrm{H} \varepsilon$ chemical shift. This might be indicative for it being involved in interaction, likely with the membrane, since all three arginines are predicted to be located at the membrane interface, at the beginning or the end of the cytosolic loop. One can notice a certain distribution of linewidths and intensities of the peaks in the spectrum, likely reflecting different structure/order and/or dynamics in different parts, as transmembrane or turns, of the protein. Uncovering these features will be subject of our further steps. 

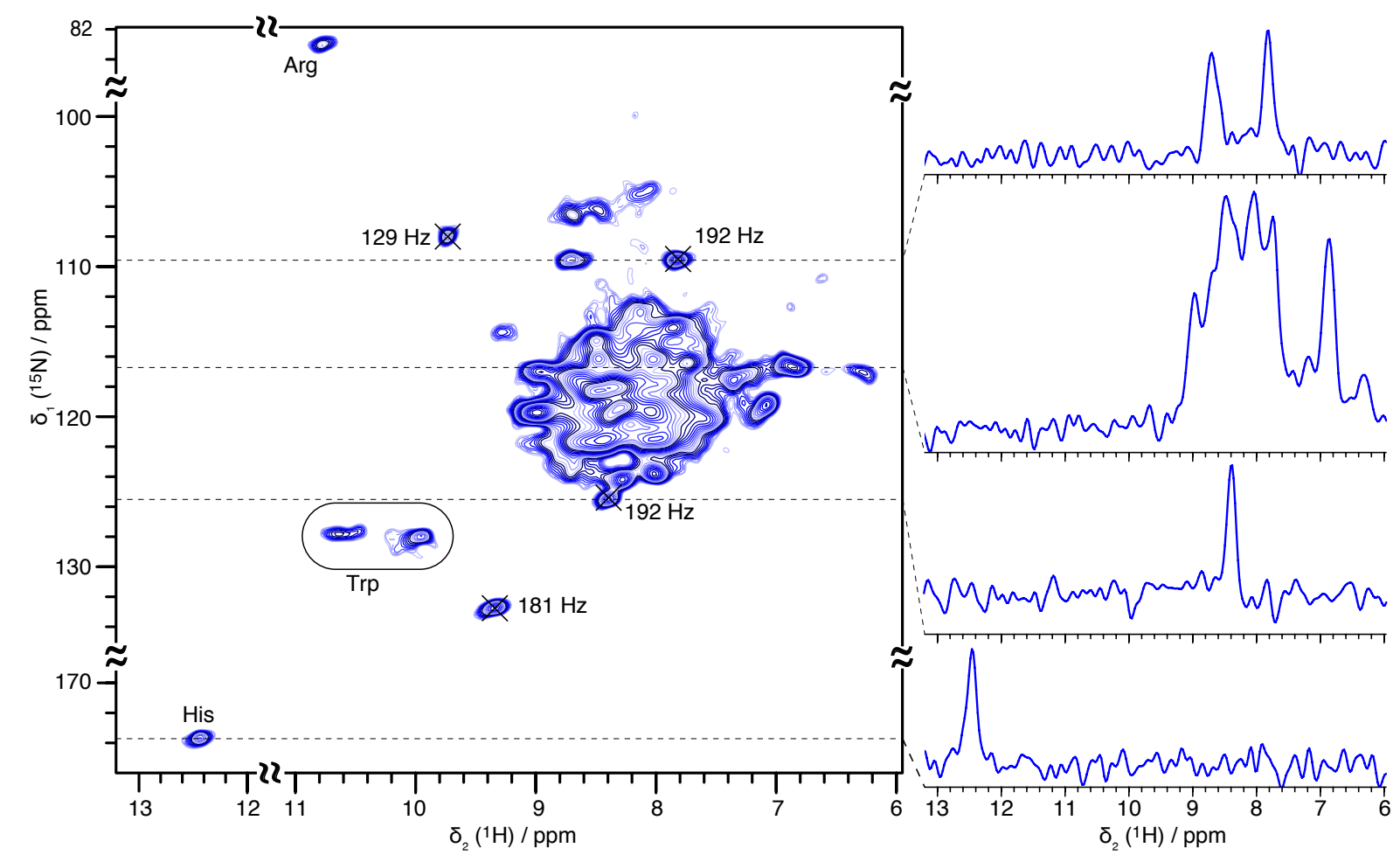

Figure 3. $2 \mathrm{D}{ }^{1} \mathrm{H}^{-15} \mathrm{~N}$ spectrum of DHBs WT at $110 \mathrm{kHz}$ MAS and $850 \mathrm{MHz}$ proton Larmor frequency. The 0.7 $\mathrm{mm}$ (outer diameter) rotor was filled using the $40 \%$ and $45 \%$ fractions of a discontinuous sucrose gradient which were directly sedimented into the rotor using a dedicated filling tool. ${ }^{[27]}$ Linewidths here have been measured on spectra processed with pure cosine waves as apodization function (SSB $=2$ in BRUKER Topspin), and are given for a selection of four signals likely presenting single resonances. The 2D spectrum has been processed using a $60^{\circ}$ shifted sinebell function ( $\mathrm{SSB}=3$ in BRUKER Topspin), and is plotted just above noise level. Figure S6 shows the spectrum with other apodization functions, in order to illustrate their effect on the resolution observed in the $2 \mathrm{D}$ plots. $1 \mathrm{D}$ traces show extracts of the $2 \mathrm{D}$ plot to illustrate $\mathrm{S} / \mathrm{N}$ ratio.

We have shown here that DHBs S can be successfully expressed in wheat germ cell-free reactions in an isotope labeled form, and that the assembly of the protein into subviral particles is spontaneous in the presence of the mild detergent MNG-3. Cell-free expressed DHBs S particles show a rather narrow distribution of diameters, close to the ones formed in vivo by the human virus $\mathrm{HBs} S,{ }^{[33]}$ and their constituent subunits display mainly $\alpha$-helical secondary structure. They show similar antigenic properties as envelopes and SVPs isolated from DHBV-infected ducks. The $2 \mathrm{D}{ }^{1} \mathrm{H}^{-15} \mathrm{~N}$ NMR spectra recorded on different preparations served to screen sample quality, and spectra from optimized preparations show sensitivity and resolution that allows to initiate structural studies. With the obtained spectra, and using 
selective labeling strategies as easily achieved in cell-free reactions, sequential assignments of the protein and subsequent structure determination get into the range of the possible, either using 3D spectroscopy, or alternatively using approaches combining defined selective labeling schemes with 2D spectroscopy. ${ }^{[34]}$ Indeed, as described in the literature, ${ }^{[35]}$ and also shown by us recently on the HCV NS4B protein, ${ }^{[17]}$ virtually clean selective carbon-13 labeling can be achieved in cell-free systems. These approaches thus shall open the way to structure investigations of HBs $\mathrm{S}$ from the duck and ultimately the human virus, and eventually enable to elucidate the structural features of complex membrane protein assemblies like these viral surface proteins, central in infection.

\section{Acknowledgements}

This work was supported by the Swiss National Science Foundation (Grant 200020_159707), the French ANR (ANR-14-CE09-0024B), and the LABEX ECOFECT (ANR-11-LABX0048) within the Université de Lyon program Investissements d'Avenir (ANR-11-IDEX0007). Electron microscopy images were recorded at the IBISA CIQLE platform (CNRS UMS3453 / INSERM US7) and we thank Elisabeth Errazuriz, Bruno Chapuis and Denis Ressnikoff for technical assistance. CD spectra were recorded at the Protein Science Platform SFB UMS 3444 / US8. R.B. was supported by the Deutsche Forschungsgemeinschaft, TRR83,

TP13 ; LL was supported by the Marie Skłodowska-Curie program (H2020-MSCA-IF-2016 748516); BHM was supported by the European Research Council (ERC grant agreement $\mathrm{n}^{\circ}$ 741863, FASTER); and $\mathrm{MN}$ by the Deutsche Forschungsgemeinschaft (NA154/9-4)

\section{References}


[1] G. R. Dreesman, F. B. Hollinger, J. L. Melnick, Appl Microbiol 1972, 24, 1001-1002.

[2] C. Seeger, F. Zoulim, W. S. Mason, Hepadnaviruses, Fields Virology, 2007.

[3] V. J. Greiner, C. Egelé, S. Oncul, F. Ronzon, C. Manin, A. Klymchenko, Y. Mély, Biochimie 2010, 92, 994-1002.

[4] P. Valenzuela, A. Medina, W. J. Rutter, G. Ammerer, B. D. Hall, Nature 1982, 298, 347-350.

[5] H. J. Stirk, J. M. Thornton, C. R. Howard, Intervirology 1992, 33, 148-158.

[6] S. Seitz, S. Urban, C. Antoni, B. Böttcher, EMBO J 2007, 26, 4160-4167.

[7] B. E. Eble, V. R. Lingappa, D. Ganem, Mol. Cell. Biol. 1986, 6, 1454-1463.

[8] K. Takai, T. Sawasaki, Y. Endo, Nat Protoc 2010, 5, 227-238.

[9] M.-L. Fogeron, A. Badillo, F. Penin, A. Böckmann, Methods Mol. Biol. 2017, 1635, 91-108.

[10] U. Schultz, E. Grgacic, M. Nassal, Adv. Virus Res. 2004, 63, 1-70.

[11] R. Zhang, K. H. Mroue, A. Ramamoorthy, Acc. Chem. Res. 2017, 50, 1105-1113.

[12] Y. Ishii, A. Wickramasinghe, I. Matsuda, Y. Endo, Y. Ishii, Y. Nishiyama, T. Nemoto, T. Kamihara, J. Magn. Reson. 2017, 286, 99-109.

[13] R. Linser, Solid State NMR 2017, 87, 45-53.

[14] D. Lalli, M. N. Idso, L. B. Andreas, S. Hussain, N. Baxter, S. Han, B. F. Chmelka, G. Pintacuda, J. Am. Chem. Soc. 2017, 139, 13006-13012.

[15] J. Struppe, C. M. Quinn, M. Lu, M. Wang, G. Hou, X. Lu, J. Kraus, L. B. Andreas, J. Stanek, D. Lalli, et al., Solid State NMR 2017, 87, 117-125.

[16] A. Laguerre, F. Löhr, F. Bernhard, V. Dötsch, Methods in Enzymology 2015, 565, 367-388.

[17] M.-L. Fogeron, V. Jirasko, S. Penzel, D. Paul, R. Montserret, C. Danis, D. Lacabanne, A. Badillo, J. Gouttenoire, D. Moradpour, et al., J. Biomol. NMR 2016, 65, 87-98.

[18] T. Terada, S. Yokoyama, Methods in Enzymology 2015, 565, 311-345.

[19] M. Wang, C. M. Quinn, J. R. Perilla, H. Zhang, R. Shirra, G. Hou, I.-J. Byeon, C. L. Suiter, S. Ablan, E. Urano, et al., Nature Com 2017, 8, 1779.

[20] A. Mainz, T. L. Religa, R. Sprangers, R. Linser, L. E. Kay, B. Reif, Angew. Chem. Int. Ed. 2013, 52, 8746-8751.

[21] S. Wang, V. Ladizhansky, Progr NMR Spectr 2014, 82, 1-26.

[22] H. Kaur, A. Lakatos-Karoly, R. Vogel, A. Nöll, R. Tampé, C. Glaubitz, Nature Com 2016, 7, 1386413876.

[23] M.-L. Fogeron, A. Badillo, V. Jirasko, J. Gouttenoire, D. Paul, L. Lancien, D. Moradpour, R. Bartenschlager, B. H. Meier, F. Penin, et al., Protein Expr Purif 2015, 105, 39-46.

[24] W. S. Mason, G. Seal, J. Summers, J. Virol. 1980, 36, 829-836.

[25] M.-L. Fogeron, D. Paul, V. Jirasko, R. Montserret, D. Lacabanne, J. Molle, A. Badillo, C. Boukadida, S. Georgeault, P. Roingeard, et al., Protein Expr Purif 2015, 1-21.

[26] J. C. Pugh, Q. Di, W. S. Mason, H. Simmons, J. Virol. 1995, 69, 4814-4822.

[27] A. Böckmann, C. Gardiennet, R. Verel, A. Hunkeler, A. Loquet, G. Pintacuda, L. Emsley, B. H. Meier, A. Lesage, J. Biomol. NMR 2009, 45, 319-327.

[28] S. Penzel, A. A. Smith, V. Agarwal, A. Hunkeler, M.-L. Org, A. Samoson, A. Böckmann, M. Ernst, B. H. Meier, J. Biomol. NMR 2015, 63, 165-186.

[29] E. Barbet-Massin, A. J. Pell, J. S. Retel, L. B. Andreas, K. Jaudzems, W. T. Franks, A. J. Nieuwkoop, M. Hiller, V. Higman, P. Guerry, et al., J. Am. Chem. Soc. 2014, 136, 12489-12497.

[30] S. Wang, S. Parthasarathy, Y. Xiao, Y. Nishiyama, F. Long, I. Matsuda, Y. Endo, T. Nemoto, K. Yamauchi, T. Asakura, et al., Chem. Commun. (Camb.) 2015, 51, 15055-15058.

[31] S. Reckel, D. Gottstein, J. Stehle, F. Löhr, M.-K. Verhoefen, M. Takeda, R. Silvers, M. Kainosho, C. 
Glaubitz, J. Wachtveitl, et al., Angew. Chem. Int. Ed. 2011, 50, 11942-11946.

[32] F. Hagn, M. Etzkorn, T. Raschle, G. Wagner, J. Am. Chem. Soc. 2013, 135, 1919-1925.

[33] M. Zahid, H. Lünsdorf, U. Rinas, Vaccine 2015, 33, 3739-3745.

[34] C. Klammt, I. Maslennikov, M. Bayrhuber, C. Eichmann, N. Vajpai, E. J. C. Chiu, K. Y. Blain, L. Esquivies, J. H. J. Kwon, B. Balana, et al., Nat Meth 2012, 9, 834-839.

[35] M. Takeda, M. Kainosho, Adv. Exp. Med. Biol. 2012, 992, 167-177. 


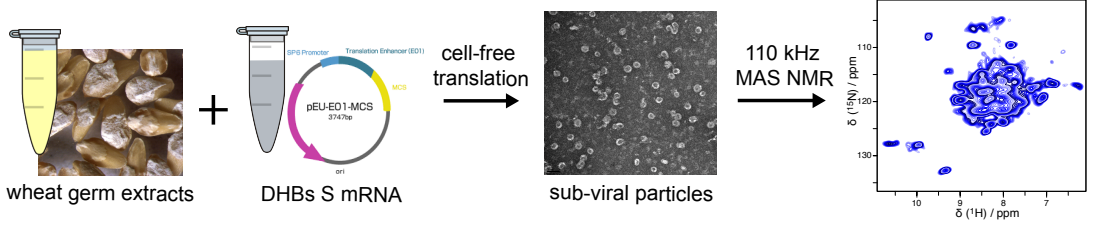

A combination of cell-free protein expression and $110 \mathrm{kHz}$ magic-angle spinning solidstate NMR allows to obtain spectra of less than $500 \mu \mathrm{g}$ in-vitro self-assembled sub-viral particles of the duck hepatitis B virus. This should pave the way for investigations of this kind of large membrane protein assemblies which structures remained intractable.
Guillaume David, Marie-Laure Fogeron, Maarten Schledorn, Roland Montserret, Uta Haselmann, Susanne Penzel, Aurélie Badillo, Lauriane Lecoq, Patrice André, Michael Nassal, Ralf Bartenschlager, Beat $H$. Meier* \& Anja Böckmann*

Page No. - Page No.

Towards structural studies of self-assembled subviral particles: combining cell-free expression with $110 \mathrm{kHz}$ MAS NMR 